\title{
Low-to-high confinement transition mediated by turbulence radial wave number spectral shift in a fusion plasma
}

Xu, G. S.; Wan, B. N.; Wang, H. Q.; Guo, H. Y.; Naulin, Volker; Rasmussen, Jens Juul; Nielsen, Anders Henry; Wu, X. Q.; Yan, Ning; Chen, L.

Total number of authors:

14

Published in:

Physical Review Letters

Link to article, DOI:

10.1103/PhysRevLett.116.095002

Publication date:

2016

Document Version

Publisher's PDF, also known as Version of record

Link back to DTU Orbit

Citation (APA):

Xu, G. S., Wan, B. N., Wang, H. Q., Guo, H. Y., Naulin, V., Rasmussen, J. J., Nielsen, A. H., Wu, X. Q., Yan, N., Chen, L., Shao, L. M., Chen, R., Wang, L., \& Zhang, W. (2016). Low-to-high confinement transition mediated by turbulence radial wave number spectral shift in a fusion plasma. Physical Review Letters, 116(9), 095002. https://doi.org/10.1103/PhysRevLett.116.095002

\section{General rights}

Copyright and moral rights for the publications made accessible in the public portal are retained by the authors and/or other copyright owners and it is a condition of accessing publications that users recognise and abide by the legal requirements associated with these rights.

- Users may download and print one copy of any publication from the public portal for the purpose of private study or research.

- You may not further distribute the material or use it for any profit-making activity or commercial gain

- You may freely distribute the URL identifying the publication in the public portal 


\title{
Low-to-High Confinement Transition Mediated by Turbulence Radial Wave Number Spectral Shift in a Fusion Plasma
}

\author{
G. S. Xu, ${ }^{1,}{ }^{*}$ B. N. Wan, ${ }^{1}$ H. Q. Wang, ${ }^{1}$ H. Y. Guo, ${ }^{2,1}$ V. Naulin, ${ }^{3}$ J. Juul Rasmussen, ${ }^{3}$ A. H. Nielsen, ${ }^{3}$ X. Q. Wu, ${ }^{1}$ \\ N. Yan, ${ }^{1,3}$ L. Chen, ${ }^{1}$ L. M. Shao, ${ }^{1}$ R. Chen, ${ }^{1}$ L. Wang, ${ }^{1}$ and W. Zhang ${ }^{1}$ \\ ${ }^{1}$ Institute of Plasma Physics, Chinese Academy of Sciences, Hefei 230031, People's Republic of China \\ ${ }^{2}$ General Atomics, P.O. Box 85608, San Diego, California 92186-5608, USA \\ ${ }^{3}$ PPFE, Department of Physics, Technical University of Denmark, DK 2800 Kongens Lyngby, Denmark
} (Received 22 April 2014; revised manuscript received 25 December 2015; published 1 March 2016)

\begin{abstract}
A new model for the low-to-high $(L-H)$ confinement transition has been developed based on a new paradigm for turbulence suppression by velocity shear [G. M. Staebler et al., Phys. Rev. Lett. 110, 055003 (2013)]. The model indicates that the $L-H$ transition can be mediated by a shift in the radial wave number spectrum of turbulence, as evidenced here, for the first time, by the direct observation of a turbulence radial wave number spectral shift and turbulence structure tilting prior to the $L-H$ transition at tokamak edge by direct probing. This new mechanism does not require a pretransition overshoot in the turbulent Reynolds stress, shunting turbulence energy to zonal flows for turbulence suppression as demonstrated in the experiment.
\end{abstract}

DOI: 10.1103/PhysRevLett.116.095002

The mechanism for the low-to-high $(L-H)$ confinement transition in toroidal magnetic confinement systems is a long-standing mystery and of great importance for understanding the physics basis for fusion energy [1]. There has been a large body of work in the past few years, focusing on slow $L-H$ transitions near the power threshold, where a transitory intermediate oscillatory phase can be identified [2-11]. A model based on turbulence-driven zonal flows has been proposed to explain the oscillatory behavior and transition trigger $[12,13]$. This model predicts a pretransition overshoot in the turbulent Reynolds stress with energy transfer from turbulence to zonal flows, so that zonal flows are driven to sufficiently high amplitude that turbulence is suppressed, reducing thermal transport and increasing the ion pressure gradient at plasma edge. Eventually, the ion pressure gradient by itself is large enough so that the equilibrium $\mathbf{E} \times \mathbf{B}$ shear, i.e., mean flow shear, can suppress the turbulence and the plasma transitions to an $H$ mode. Some supporting experimental evidence has been reported recently [2-9]. However, there are still some observations that cannot be interpreted in terms of zonalflow-based transition theory [10,11]. A question raised by this body of work is whether normal, single-step, fast $L-H$ transitions merely compress the oscillatory behavior into a short time, i.e., just one cycle of the oscillation with the Reynolds stress overshot behavior or whether the process is skipped entirely and a different process occurs.

One central issue for the $L-H$ transition is what leads to the turbulence suppression in such short time scale. Shunting turbulence energy to zonal flows offers one possible explanation. Given the diverse experimental observations, it is, in fact, reasonable to suspect that there might be multiple mechanisms at play [14]. More recently, a different $L-H$ transition mechanism based on a turbulence radial wave number spectral shift has been proposed [15-18], where the turbulence suppression at the transition is due to breaking down of the ballooning symmetry by velocity shear in a toroidal system, which leads to a shift in the radial wave number spectrum of turbulence, tilts the turbulence eddies, and scatters the spectral energy to higher $k_{\perp}$ (perpendicular wave number) region where the energy is damped, since the viscous dissipation scales with $k_{\perp}^{2}$ [15]. This new mechanism does not need to involve zonal flows or Reynolds stress.

In this Letter, a new $L-H$ transition model has been developed based on the new turbulence suppression mechanism [15], which demonstrates that the fast transition can occur spontaneously mediated by a shift in the radial wave number spectrum of turbulence without the assistance of zonal flows, as evidenced here, for the first time, by the direct observation of a turbulence radial wave number spectral shift and turbulence structure tilting prior to and across the $L-H$ transition at the plasma edge in the EAST superconducting tokamak using a $3 \times 4$ probe array. This new finding may shed light on the recent experimental observations concerning the $L-H$ transition process [14], which appear to be inconsistent with the previous model based on turbulence-driven zonal flows.

The new model consists of two coupled equations:

$$
\begin{gathered}
\partial \Phi / \partial t=\gamma_{k_{y}} \Phi+\gamma_{\text {shear }} k_{y} \partial \Phi / \partial k_{x} \\
-\left(c_{y} k_{y}^{2}+c_{x} k_{x}^{2}\right) \Phi^{2}+D \partial^{2} \Phi / \partial k_{x}^{2}, \\
\partial|\nabla p| / \partial t=Q-\left(\chi+\chi_{\text {neo }}\right)|\nabla p| .
\end{gathered}
$$

This is a time-dependent one-dimensional (in $k_{x}$ space) model. All parameters in the model use the typical values at 
the EAST tokamak edge based on realistic experimental conditions for quantitative comparison with the experiments. The shift in the $k_{x}$ spectrum induced by the velocity shear is modeled with an analytic nonlinear Bernoulli differential equation [Eq. (1)], as proposed in Ref. [15]. Here, $\Phi=\left\langle\left|e \tilde{\phi}_{k_{y}, k_{x}} / T_{e}\right|^{2}\right\rangle^{1 / 2}$ is the time and magnetic fluxsurface-averaged Fourier amplitude of the electric potential fluctuations normalized to the local electron temperature $\left(T_{e}=100 \mathrm{eV}\right)$ at a fixed poloidal $k_{y}=k_{\theta} \rho_{s}$ and radial $k_{x}=k_{r} \rho_{s} \quad$ wave number, where $\rho_{s}=c_{s} / \Omega_{c}, \quad c_{s}=$ $\sqrt{T_{e} / m_{i}}=69 \mathrm{~km} / \mathrm{s}$ is the sound speed at plasma edge for deuterium plasmas, $\Omega_{c}=e B / m_{i}, B=2 T, \theta$ is the poloidal angle, and $r$ is the minor radius. $k_{y}$ is fixed at 0.1 , which is a typical value at the EAST plasma edge. The time unit in the model is $\tau=a / c_{s}=6.5 \mu$ s where $a=0.45 \mathrm{~m}$ is the minor radius of EAST plasma.

Equation (1) describes the nonlinear evolution of the amplitude of the electric potential fluctuations. The second term on the right-hand side describes the shift in the radial wave number spectrum induced by the shear in fluctuation phase velocity $v_{\text {ph }}$ (mainly in the poloidal direction) in the lab frame, the so-called "Doppler shift" [19]. In the geometric space, it reflects the tilting of the global envelope structure of the toroidal eigenmodes by velocity shear [20]. $\gamma_{\text {shear }}$ is the velocity shearing rate defined by $\gamma_{\text {shear }}=$ $(r / q) \partial\left(q v_{\mathrm{ph}} / r\right) / \partial r$ [19], where $q$ is the safety factor, and $v_{\mathrm{ph}}$ is composed of $\mathbf{E} \times \mathbf{B}$ drift velocity $v_{E \times B}$ and phase velocity in the plasma frame $v_{\text {php }} . v_{E \times B}$ is related to the ion pressure gradient and ion perpendicular fluid velocity through the radial force balance equation, $v_{E \times B}=\nabla p_{i} /$ $e B n_{i}+v_{i \perp} \cdot v_{E \times B}$ can be generated spontaneously at the tokamak edge through several mechanisms, such as a steepening of the ion pressure gradient, ion orbit loss, plasma rotation, or turbulence-driven momentum transport via turbulent Reynolds stress [12]. Even though there is no change in $v_{E \times B}$, some changes in $v_{\text {php }}$ due to some modifications in the turbulence modes can also lead to $v_{\text {ph }}$ change.

$\gamma_{k_{y}}$ is the linear growth rate of the most unstable mode at $k_{y}=0.1$. It depends on the ballooning angle of the most unstable mode, $\gamma_{k_{y}}=\gamma_{0} \cos \left(\theta_{0 \max }\right)$ [20], where $\gamma_{0}=$ $\gamma|\nabla p|^{1 / 2}$ is the normalized maximum linear growth rate for pressure-driven ballooning-type instabilities without velocity shear. The growth rate $\gamma_{0} / \tau=c_{s}\left(2 / R L_{p}\right)^{1 / 2} \sim$ $0.7 \mathrm{MHz}$ is close to the spectral bandwidth of the $L$-mode edge turbulence on EAST. Here, $\gamma=a(2 / p R)^{1 / 2}=4, R=$ $1.9 \mathrm{~m}$ is the major radius of EAST, $p$ is the plasma pressure, $\nabla p$ is the edge pressure gradient, and $L_{p}=p /|\nabla p|=$ $\left(a / \gamma_{0}\right)^{2} 2 / R$ is the gradient length, which is typically $\sim 1 \mathrm{~cm}$ in the EAST pedestal. $\theta_{0 \max }=\left[\delta^{1 / 3}+\right.$ $\left.\lambda \delta^{4} \arctan (\delta)\right]\left(1+\lambda \delta^{4}\right)^{-1}$ is the approximate solution to the difference equation $\partial\left[\cos \left(\theta_{0}\right)-\delta /\left|\sin \left(\theta_{0}\right)\right|\right] /$ $\partial \theta_{0}=0$ [20], where $\lambda=0.0938$ gives the best fit to the solution, $\delta=\gamma_{\text {shear }} / 2 \hat{s} \gamma_{0}$ is the normalized velocity shear parameter, and $\hat{s}=r d q / q d r=2$ is the magnetic shear parameter at the plasma edge. With increasing velocity shear, the ballooning angle $\theta_{0 \max }$ shifts away from the outboard midplane $(\theta=0)$ towards $\theta= \pm \pi / 2$, leading to a reduced maximum linear growth rate located at the poloidal angle $\theta \sim \theta_{0 \max }$ and the tilting of the global envelope structure of the toroidal eigenmodes with the tilt angle for the most unstable mode roughly at $\theta_{0 \max }$ [20]. If $\gamma_{\text {shear }}$ is dominated by $\mathbf{E} \times \mathbf{B}$ shear, it can be tied to the ion pressure gradient according to the radial force balance equation, $\gamma_{\text {shear }}=\alpha|\nabla p|$, where $\alpha=0.837$. With this coefficient, $\gamma_{\text {shear }} / \tau \sim 0.2 \mathrm{MHz}$ in $L$ mode and $\sim 0.5 \mathrm{MHz}$ in $H$ mode, which are typical values at the EAST plasma edge.

The third term is a quadratic nonlinear term, which represents the effect of nonlinear mode coupling and viscous dissipation, leading to turbulence damping. Since the actual nonlinear mode coupling term in the gyrokinetic equation is a convolution over the $k_{x}$ space [15], we add a diffusion term, i.e., the fourth term with $D=0.001$, to recover the mode coupling between adjacent $k_{x}$. The coefficient $c_{y}$ determines the strength of dissipation and, thus, the time scale for turbulence suppression at the $L-H$ transition. Here, $c_{y}=1000$ to allow turbulence suppression within $0.5 \mathrm{~ms}$. The anisotropy ratio is fixed at $c_{x} / c_{y}=0.56$, which gives the best fits to the spectra from GYRO code simulations [15]. The spectral shift term (second term) is actually an advection term in $k_{x}$ space. It shifts the spectral energy towards higher $k_{x}$, which enhances the dissipation through the third term, since the viscous dissipation scales with $k_{x}^{2}$.

Equation (2) describes cross-field thermal transport at plasma edge. $Q$ is the heat flux from the plasma core, which is proportional to input heating power. $\chi_{\text {neo }}=5 \times 10^{-4}$ is the neoclassical thermal conductivity. $\chi=\left(c_{0} / 2 k_{x \max }\right) \times$ $\int_{-k_{x \max }}^{k_{x \max }} k_{y} \Phi^{2} d k_{x}$ is the turbulence-driven thermal conductivity with $c_{0}=1 . \chi \gg \chi_{\text {neo }}$ needs to be satisfied in $L$-mode plasmas. $k_{x \max }=3$ defines the $k_{x}$ range for the modeling. Most of the spectral power is contained in this range. The $k_{x}$ space is digitized into 3000 grids in this modeling.

Equations (1) and (2) are coupled through the dependence of $\gamma_{k_{y}}$ and $\gamma_{\text {shear }}$ on the edge pressure gradient $|\nabla p|$. The zerospectral-flux boundary condition in the $k_{x}$ space has been used, i.e., $\Gamma_{k x}=\gamma_{\text {shear }} k_{y} \Phi+D \partial \Phi / \partial k_{x}=0$, to ensure that no spectral energy flows into or out of the turbulence system through the left or right boundaries. The only way for energy coming into the system is through the linear growth term, i.e., the first term of Eq. (1), and going out through the nonlinear mode coupling and viscous dissipation term, i.e., the third term of Eq. (1). The initial conditions for the modeling are $|\nabla p|=1$ and $\Phi=\Phi_{0} c_{y} k_{y}^{2} /\left(c_{y} k_{y}^{2}+c_{x} k_{x}^{2}\right)$. The initial spectrum is symmetric with respect to the sign of $k_{x}$ with spectral peak $\Phi_{0}=35 \%$.

Figure 1 shows the modeling results with time evolution for 25000 time units, corresponding to $162.5 \mathrm{~ms}$ in total. The $L-H$ transition occurs at $84 \mathrm{~ms}$, which is controlled by the input heating power $Q$. The modeling starts with low 


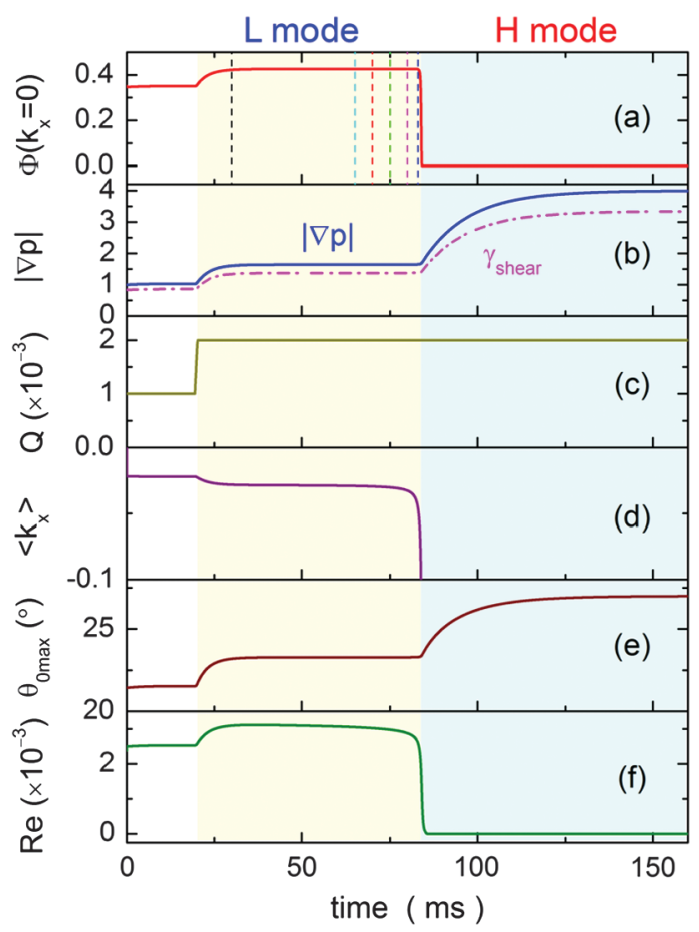

FIG. 1. Time evolution of (a) turbulence amplitude at $k_{x}=0$, (b) edge pressure gradient and shearing rate of phase velocity, (c) heat flux from the plasma core, (d) spectral-averaged $k_{x}$, (e) ballooning angle in degrees for the maximum growth rate mode, and (f) turbulent Reynolds stress.

power, $Q=0.001$. The system reaches an $L$-mode stationary state after $10 \mathrm{~ms}$, which is still far below the transition power threshold. At $t=20 \mathrm{~ms}$, the input heating power is doubled. At this power level, the plasma still stays in the $L$ mode but evolves slowly towards the transition point. The edge pressure gradient $|\nabla p|$, turbulence amplitude $\Phi$ at $k_{x}=0$, and ballooning angle of the most unstable mode $\theta_{0 \max }$ nearly saturate. However, during this time, the $k_{x}$ spectrum is still continuously evolving towards negative direction as approaching the transition point, as indicated by the time evolution of the spectral-averaged radial wave number $\left\langle k_{x}\right\rangle=\int_{-k_{x \max }}^{k_{\max }} \Phi^{2} k_{x} d k_{x} / \int_{-k_{x \max }}^{k_{x \max }} \Phi^{2} d k_{x}$ in Fig. 1(d). The physics direction of negative $k_{x}$ is radially outward, which is the same as that in the experiments. The $k_{x}$ spectra at several time points [marked in Fig. 1(a) by the dashed vertical lines with corresponding colors, as well as the inset in Fig. 2(a)] are shown in Fig. 2(a). The spectral change appears to mainly occur on the positive $k_{x}$ side where the spectral energy is progressively exhausted, since the spectral energy is advected towards the left, and there is no replenishment from the right end.

Near the transition point, the spectral shift is significantly accelerated, as shown in Figs. 1(d) and 2(b). At the same time, the turbulence amplitude $\Phi$ is quenched within a few hundreds of $\mu \mathrm{s}$. The quick suppression is mainly due to the nonlinear mode coupling and viscous dissipation through the third term of Eq. (1). Since the velocity shearing rate $\gamma_{\text {shear }}$ is tied to the edge pressure gradient $|\nabla p|$, its time

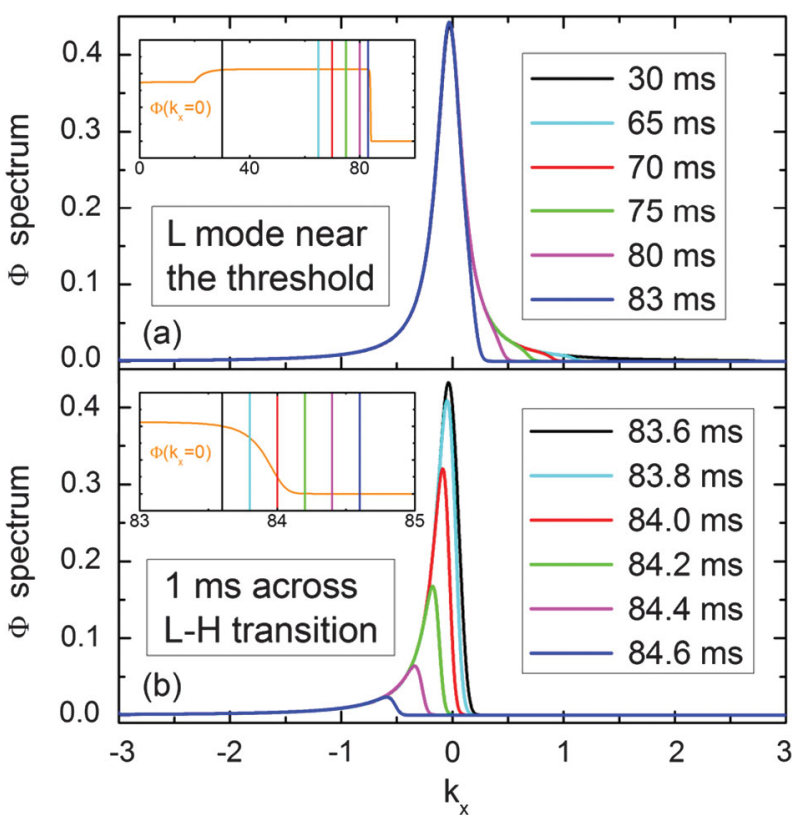

FIG. 2. The $k_{x}$ spectra at several time points with corresponding colors as indicated in the insets, (a) in the $L$ mode near the power threshold, (b) within $1 \mathrm{~ms}$ across the $L-H$ transition.

evolution follows $|\nabla p|$, as shown in Fig. 1(b). They evolve on a much slower time scale (tens of ms) with respect to the turbulence suppression or spectral shift, as normally seen in the experiments. Therefore, it appears that the fast $L-H$ transition process occurs spontaneously in the modeling, activated at a critical point of the spectral energy depletion on the positive $k_{x}$ side, and does not need to be triggered or preceded by a sudden increase in the mean flow shear.

The turbulent Reynolds stress is defined following the quasilinear formula, $\operatorname{Re}=\int_{-k_{x \max }}^{k_{x \max }} k_{y} \Phi^{2} d k_{x}$ [15]. As shown in Fig. 1(f), it does not exhibit a pretransition overshot, but, instead, slightly decreases as approaching the transition point and then quickly suppressed when the turbulence is quenched. After the transition, $|\nabla p|$ builds up on a slow time scale, $\tau / \chi_{\text {neo }} \sim 13 \mathrm{~ms}$, and finally saturates in a stationary $H$ mode with $|\nabla p|=Q / \chi_{\text {neo }}=4$, solely determined by the neoclassical transport.

Direct evidence for the turbulence radial wave number spectral shift and turbulence structure tilting prior to and across the $L-H$ transition has newly been obtained at plasma edge in EAST using a $3 \times 4$ probe array. The probe array has four layers of tips spaced radially by $\Delta r=2.5 \mathrm{~mm}$ with three tips spaced poloidally by $6 \mathrm{~mm}$ for each layer $[9,10]$, mounted on a reciprocating probe system at the outboard midplane. The tips in the first and third layers are operated as triple probes providing the measurements of $T_{e}, n_{e}$, and plasma potential $\phi_{p}=\phi_{f}+2.5 T_{e}$. The other tips are used to measure floating potentials $\phi_{f}$. The data were sampled at $1 \mathrm{MHz}$.

An typical fast $L-H$ transition with favorable ion $\nabla B$ drift direction [9] was captured by the probe array with the first layer of tips located at $\sim 5 \mathrm{~mm}$ inside the separatrix and the third layer located approximately at the separatrix. The 
electron temperatures are $T_{e 1} \sim 80 \mathrm{eV}$ and $T_{e 2} \sim 50 \mathrm{eV}$. The electron densities are $n_{e 1} \sim 1.1 \times 10^{19} \mathrm{~m}^{-3}$ and $n_{e 2} \sim 0.7 \times 10^{19} \mathrm{~m}^{-3}$ in $L$ mode just before the transition with subscripts 1 and 2 denoting the first and third layer of tips, respectively. Figure 3(a) shows the turbulence $k_{r}$ spectrum obtained from the two radially spaced floating potentials based on the standard two-point cross-correlation technique [21]. Here, the spectrum is estimated from fluctuations in $40-100 \mathrm{kHz}$, where most of the turbulence spectral power is located. The two-point cross-correlation technique is valid when the radial distance between the two points $(\Delta r=2.5 \mathrm{~mm})$ is less than the radial correlation length of turbulence, which exceeds $5 \mathrm{~mm}$ in this experiment.

The transition is indicated by a sharp drop in the divertor $D \alpha$ emission level [Fig. 3(b)] and turbulence spectral

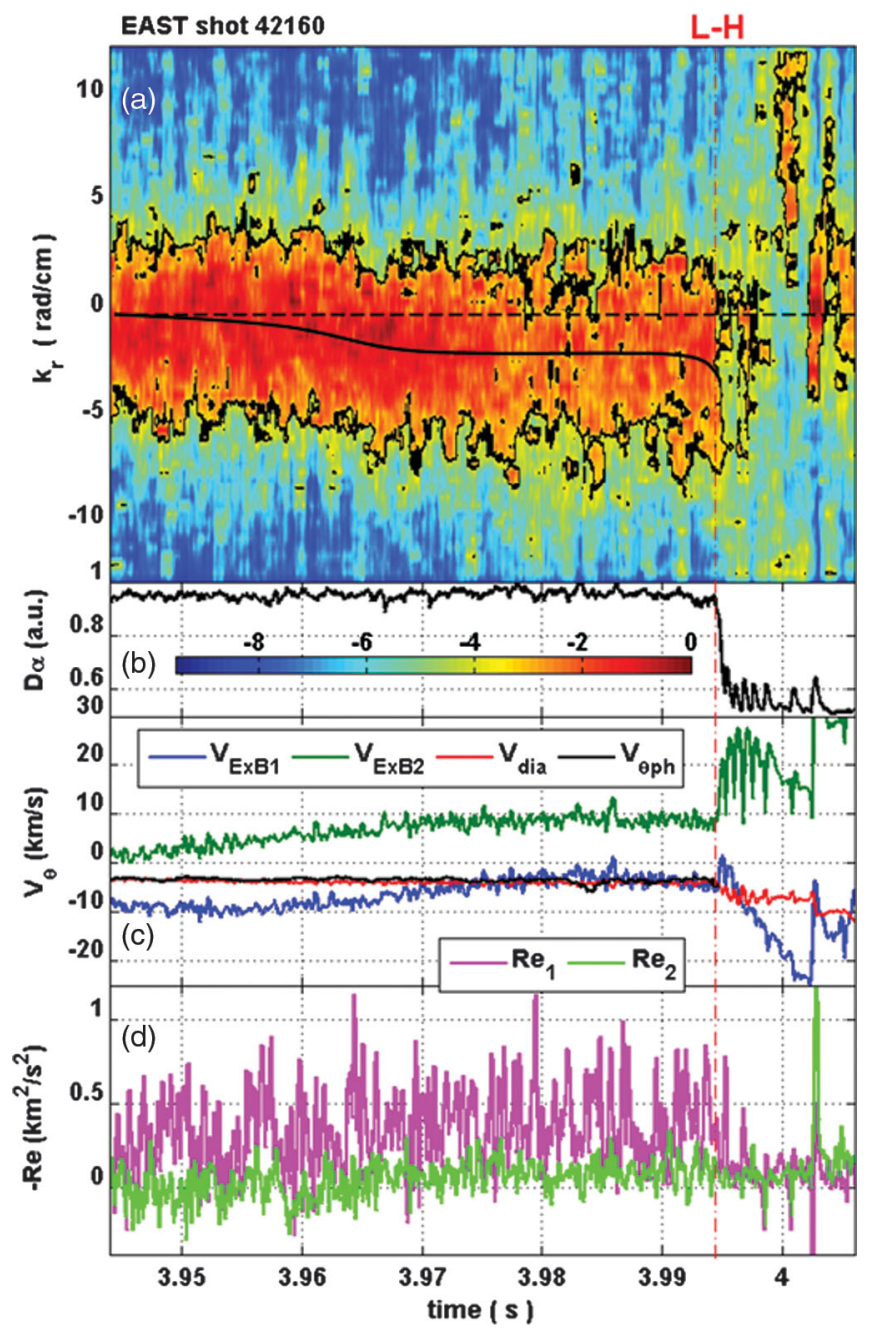

FIG. 3. Time evolution of (a) radial wave number spectrum displayed in log scale, spectral-averaged radial wave number $\left\langle k_{r}\right\rangle$ as indicated by black curve, (b) divertor $D \alpha$ emission, (c) $\mathbf{E}_{\mathbf{r}} \times \mathbf{B}$ velocity $V_{E \times B}$ at two radial locations, diamagnetic drift velocity $V_{\text {dia }}$ and poloidal phase velocity of fluctuations $V_{\theta \text { ph }}$ with negative velocity standing for the electron diamagnetic direction, (d) turbulent Reynolds stress at $\sim 5 \mathrm{~mm}$ inside the separatrix for $\mathrm{Re}_{1}$ and approximately on the separatrix for $\mathrm{Re}_{2}$. amplitude at $3.994 \mathrm{~s}$. As shown in Fig. 3(a), the $k_{r}$ spectrum appears to progressively shift towards the negative direction in the last tens of ms before the transition. Here, negative $k_{r}$ means pointing radially outwards. The change in the spectral shape is mainly manifested by a depletion of the spectral power on the positive $k_{r}$ side, consistent with the modeling results [Fig. 2(a)]. Note that a nonzero $\left\langle k_{r}\right\rangle$ extracted from the two-point cross-correlation technique, does not necessarily mean that the modes propagate radially. It could be a projection of the poloidal propagation due to the tilting of turbulence structures. The sign of $\left\langle k_{r}\right\rangle$ depends on the direction of turbulence eddy tilting, which is controlled by the direction of velocity shear and fluctuation poloidal propagation. In experiments, $\left\langle k_{r}\right\rangle$ points radially outwards in most cases at the outboard midplane, slightly inside the separatrix, because there is usually a negative $E_{r}$ well, and fluctuations propagate poloidally in the electron diamagnetic direction in the lab frame.

Figure 3(c) shows diamagnetic drift velocity $V_{\text {dia }}=$ $\Delta p_{e} /\left[\Delta r Z_{\mathrm{eff}} e B\left(n_{e 1}+n_{e 2}\right)\right]$ and poloidal phase velocity of fluctuations $V_{\theta \mathrm{ph}}$ in the lab frame measured by the probe array. Here, $V_{\theta \mathrm{ph}}$ is calculated based on the time-delay estimation technique [22], $Z_{\text {eff }}=2$ is the effective charge number, and $p_{e}=n_{e} T_{e}$. $V_{\theta \mathrm{ph}}$ is very close to $V_{\mathrm{dia}}$ and keeps nearly constant as approaching the transition, indicating that the edge pressure gradient and poloidal phase velocity does not vary much before the transition, consistent with the modeling results as shown in Fig. 1(b).

Figure 3(c) also shows the $\mathbf{E}_{\mathbf{r}} \times \mathbf{B}$ velocities at two radial locations spaced radially by $\Delta r=2.5 \mathrm{~mm}$, $V_{E \times B 1}=-\left(\Delta \phi_{f 1} / \Delta r+2.5 \Delta T_{e} / 2 \Delta r\right) / B$, and $V_{E \times B 2}=$ $-\left(\Delta \phi_{f 2} / \Delta r+2.5 \Delta T_{e} / 2 \Delta r\right) / B$. The radial difference $\left(V_{E \times B 2}-V_{E \times B 1}\right)$ can be used as an indicator of the $\mathbf{E} \times \mathbf{B}$ velocity shear. The $k_{r}$ spectrum shift appears to be correlated with the time evolution of $\left(V_{E \times B 2}-V_{E \times B 1}\right)$; e.g., there appears to be a clear shift event in the $k_{r}$ spectrum between 3.96 and $3.97 \mathrm{~s}$ [Fig. 3(a)]. Before this shift event $\left(V_{E \times B 2}-V_{E \times B 1}\right)$ slowly increases. After this shift event, it slightly decreases, at the same time the spectral shift slows down until the final $L-H$ transition.

Across the transition, the spectral shift is significantly accelerated, as indicated by the inclined spectral transition front marked by the purple dashed line in Fig. 4(a), which is the zoom-in plot of Fig. 3(a) near the transition point. The $k_{r}$ spectrum moves completely to the negative $k_{r}$ side; meanwhile, the turbulence level is significantly reduced within a few hundreds of $\mu \mathrm{s}$, just like those predicted by the model in Fig. 2(b). There is no significant change in $\left(V_{E \times B 2}-V_{E \times B 1}\right)$ prior to the transition, as shown in Fig. 3(c). Both $V_{E \times B 1}$ and $V_{E \times B 2}$ jump up towards the positive direction at the same time of turbulence suppression [9]. However, $V_{E \times B 2}$ appears to increase more, suggesting a prompt increase of velocity shear at the transition. The fast spectral shift across the transition appears to be a consequence of the progressive accumulation of spectral shift developed in the last tens of ms before the transition, until a critical point. 



FIG. 4. (a) Time history of $k_{r}$ spectrum near the $L-H$ transition. (b)-(d) Power spectra in $k_{r}-k_{\theta}$ space at three time points: $t=3.95 \mathrm{~s}$ (40 ms before the transition), $t=3.97 \mathrm{~s}$ (20 ms before the transition), $t=3.994 \mathrm{~s}$ (just prior to the transition).

Figure 3(d) shows the turbulent Reynolds stress measured at two radial locations spaced radially by $2 \Delta r=5 \mathrm{~mm}$, indicating the presence of significant radial gradient at plasma edge in $L$ mode. However, its amplitude does not change much with time before the transition, and there appears to be no pretransition overshoot in either the turbulent Reynolds stress or its radial gradient. On the contrary, the turbulent Reynolds stress quickly decreases across the transition, mainly due to the reduction of turbulence levels, again in agreement with the modeling results shown in Fig. 1(f). In addition, the power spectra of $V_{E \times B 1}$ are quite flat in the low frequency range $(0.1-40 \mathrm{kHz})$ prior to the transition, and no clear evidence for the existence of zonal flows can be identified from the power spectra.

Figure 4 shows the turbulence power spectra in $k_{r}-k_{\theta}$ space at three time points: (a) $t=3.95 \mathrm{~s}$ (40 ms before the transition), (b) $t=3.97 \mathrm{~s}$ (20 ms before the transition), and (c) $t=3.994 \mathrm{~s}$ (just prior to the transition), indicating that the tilting angle of the turbulence structures increases as approaching the transition point in addition to the spectral shift towards negative $k_{r}$. The spectra concentrate mostly in the negative $k_{\theta}$ space, since the turbulence propagates poloidally in the electron diamagnetic direction in the lab frame.

In summary, this Letter demonstrates, for the first time, that the normal, single-step, fast $L-H$ transition in toroidal plasmas can be mediated by a shift in the radial wave number spectrum of turbulence induced by mean flow shear without involving zonal flows, although zonal flows may still play a role in the limit-cycle oscillations during slow $L-H$ transition near the transition power threshold. According to this new turbulence suppression mechanism, i.e., scattering turbulence energy to higher $k_{\perp}$ region instead of inverse cascading to zonal flows [15], a bifurcation driven solely by the mean flow shear is possible. Furthermore, the bifurcation is not necessarily triggered or preceded by a sudden increase in the mean flow shear. The increase of mean flow shear typically occurs right after the transition as a consequence of the turbulence suppression. These new findings may help elucidate discrepancies between the recent experimental observations and the previous zonal-flow theories on the $L-H$ transition, concerning the causality between the electric field and turbulence [10] and the presence of zonal flows at the transition [11].

This work was supported by National Magnetic Confinement Fusion Science Program of China under Contracts No. 2015GB101000, No. 2013GB106000, and No. 2013GB107000 and National Natural Science Foundation of China under Contracts No. 11575235 and No. 11422546.

*Corresponding author. gsxu@ipp.ac.cn

[1] F. Wagner et al., Phys. Rev. Lett. 49, 1408 (1982).

[2] T. Estrada, C. Hidalgo, T. Happel, and P. H. Diamond, Phys. Rev. Lett. 107, 245004 (2011).

[3] G. D. Conway, C. Angioni, F. Ryter, P. Sauter, and J. Vicente, Phys. Rev. Lett. 106, 065001 (2011).

[4] G. S. Xu et al., Phys. Rev. Lett. 107, 125001 (2011).

[5] L. Schmitz, L. Zeng, T. L. Rhodes, J. C. Hillesheim, E. J. Doyle, R. J. Groebner, W. A. Peebles, K. H. Burrell, and G. Wang, Phys. Rev. Lett. 108, 155002 (2012).

[6] P. Manz et al., Phys. Plasmas 19, 072311 (2012).

[7] G. R. Tynan et al., Nucl. Fusion 53, 073053 (2013).

[8] Z. Yan, G. R. McKee, R. Fonck, P. Gohil, R. J. Groebner, and T. H. Osborne, Phys. Rev. Lett. 112, 125002 (2014).

[9] G. S. Xu et al., Nucl. Fusion 54, 103002 (2014).

[10] J. Cheng et al., Phys. Rev. Lett. 110, 265002 (2013).

[11] T. Kobayashi et al., Phys. Rev. Lett. 111, 035002 (2013).

[12] E.-J. Kim and P. H. Diamond, Phys. Rev. Lett. 90, 185006 (2003).

[13] K. Miki, P. H. Diamond, Ö. D. Gürcan, G. R. Tynan, T. Estrada, L. Schmitz, and G.S. Xu, Phys. Plasmas 19, 092306 (2012).

[14] K. Itoh, S.-I. Itoh, and A. Fujisawa, Plasma Fusion Res. 8, 1102168 (2013).

[15] G. M. Staebler, R. E. Waltz, J. Candy, and J. E. Kinsey, Phys. Rev. Lett. 110, 055003 (2013).

[16] G. M. Staebler, J. Candy, R. E. Waltz, J. E. Kinsey and W. M. Solomon , Nucl. Fusion 53, 113017 (2013).

[17] G. M. Staebler and R. J. Groebner, Plasma Phys. Controlled Fusion 57, 014025 (2015).

[18] G. M. Staebler and R. J. Groebner, Nucl. Fusion 55, 073008 (2015).

[19] R. E. Waltz, R. L. Dewar, and X. Garbet, Phys. Plasmas 5, 1784 (1998).

[20] J. Y. Kim, Y. Kishimoto, M. Wakatani, and T. Tajima, Phys. Plasmas 3, 3689 (1996).

[21] Ch. P. Ritz et al., Rev. Sci. Instrum. 59, 1739 (1988).

[22] G. R. McKee, R. J. Fonck, D. K. Gupta, D. J. Schlossberg, M. W. Shafer, C. Holland, and G. Tynan, Rev. Sci. Instrum. 75, 3490 (2004). 